\title{
Resonant Andreev reflections in superconductor-carbon-nanotube devices
}

\author{
Yadong Wei, ${ }^{1}$ Jian Wang, ${ }^{1}$ Hong Guo, ${ }^{2}$ Hatem Mehrez, ${ }^{2}$ and Christopher Roland ${ }^{3}$ \\ ${ }^{1}$ Department of Physics, The University of Hong Kong, Pokfulam Road, Hong Kong, China \\ ${ }^{2}$ Center for the Physics of Materials and Department of Physics, McGill University, Montreal, PQ, Canada H3A 2T8 \\ ${ }^{3}$ Department of Physics, The North Carolina State University, Raleigh, North Carolina 27695
}

(Received 27 November 2000; published 25 April 2001)

\begin{abstract}
Resonant Andreev reflection through superconductor-carbon-nanotube devices was investigated theoretically with a focus on the superconducting proximity effect. Consistent with a recent experiment, we find that for on-resonance high-transparency devices, the Andreev current is characterized by a large value and a resistance dip; low-transparency off-resonance devices give the opposite result. We also give evidence that the observed low-temperature transport anomaly may be a natural result of the Andreev reflection process.
\end{abstract}

DOI: 10.1103/PhysRevB.63.195412

PACS number(s): 72.80.Rj, 73.23.Ad, 73.61.Wp

The field of carbon nanotube research has recently entered a new phase with the fabrication of hybrid device structures, in which carbon nanotubes are contacted electrically with other materials. ${ }^{1-4}$ This is a crucial step, as a carbonnanotube-based microelectronics is only possible when finite nanotubes can be efficiently fabricated and coupled to external leads. Examples of such hybrid devices include nanotube-based magnetic tunnel junctions, ${ }^{1}$ nanotube heterostructure, ${ }^{2}$ and superconducting junctions., ${ }^{3,4}$ These systems not only have significant application potential in their own right, but also provide an important testing ground for fundamental physics at the nanometer length scale. Of particular interest is the important role played by the electronic structure in determining the transport properties of nanotube devices.

In this paper we report a theoretical analysis of a hybrid superconductor-nanotube junction that has been the subject of a recent experimental study. ${ }^{4}$ The experimental device consists of a single-wall metallic carbon nanotube (SWNT) bridging two superconducting electrodes. By tuning the transparency of the device, ${ }^{4}$ clear signals of Andreev reflections ${ }^{5}$ were detected via changes in the subgap resistance at a temperature of $T=4.2 \mathrm{~K}$, while other transport anomalies were observed at lower $T$. To date, there have been many theoretical and experimental studies of normal metal $(N)$ /superconductor $(S)$ interfaces on a mesoscopic scale. $^{6-19}$ However, no such analysis exists for molecular devices where the specific molecular orbitals play a important role. By combining standard nonequilibrium Green's function techniques ${ }^{9-11}$ (NEGF) with a tight-binding model (TB) for the SWNT, we have analyzed quantum transport properties of SWNT-S junctions. Our results are consistent with the experimental data. ${ }^{4}$

Although the experimental device consisted of two SWNT-S junctions, the data indicates that each of these junctions acts independently. ${ }^{4}$ Hence, we focus here on the somewhat simpler problem of a $N$-SWNT-S system, leaving an analysis of the multiple Andreev reflections of a $S$-SWNT-S system for the future. Our theory proceeds by combining the NEGF with a standard TB model for the $\mathrm{SWNT}^{20}$ such that the coupling of the nanotube to the $N$ (left) and $S$ (right) leads are included via their appropriate self-energies. By iterating the equation of $\operatorname{motion}^{21}$ (and we refer interested readers to Ref. 11 for the standard but tedious algebra $^{11}$ ), the electric current flowing through the device is found to have two contributions: i.e., $I=I_{A}+I_{1}$. The Andreev current $I_{A}$, or subgap contribution, is given by $(\hbar$ $=e=1)$ :

$$
I_{A}=\frac{1}{\pi} \int d E\left[f_{L}(E-V)-f_{L}(E+V)\right] T_{A}(E),
$$

where $f_{L, R}$ denote the Fermi functions of the left and right leads, respectively, $E$ denotes the electron energy, and $V$ denotes the bias potential. The Andreev reflection probability $T_{A}(E)$ is given by

$$
T_{A}(E) \equiv \operatorname{Tr}\left[\Gamma_{L} \mathbf{G}_{12}(E) \Gamma_{L} \mathbf{G}_{12}^{\dagger}(E)\right],
$$

where $\Gamma_{L, R}$ are the appropriate linewidth functions describing the coupling of the SWNT to the respective leads. Here $\mathbf{G}_{11}$ and $\mathbf{G}_{12}$ are the retarded Green's functions ${ }^{10,11}$ of the SWNT, which include the proper self-energies of the leads. These are evaluated by direct matrix inversion ${ }^{22}$ for tightbinding Hamiltonians. The remaining contribution to the current is given by

$$
\begin{aligned}
I_{1}= & \frac{1}{\pi} \int d E \operatorname{Tr}\left[T_{1}(E)+T_{2}(E)+T_{3}(E)\right] \\
& \times\left[f_{L}(E-V)-f_{R}(E)\right] \rho_{R}(E),
\end{aligned}
$$

where the density of states of the $S$ lead is $\rho_{R}(E)$ $=|E| / \sqrt{E^{2}-\Delta^{2}}$ for $|E|>\Delta$, and zero otherwise, and $\Delta$ is the gap energy of the superconductor. Here $T_{1}, T_{2}, T_{3}$ are transmission probabilities for different physical processes that are only nonzero when $|E| \geqslant \Delta$. Hence, these processes describe excitations of the system. In particular, $T_{1}=\Gamma_{L} \mathbf{G}_{11} \Gamma_{R} \mathbf{G}_{11}^{\dagger}$ gives the familiar tunneling current; $T_{2}=\Gamma_{L} \mathbf{G}_{12} \Gamma_{R} \mathbf{G}_{12}^{\dagger}$ denotes the branch-crossing process of Blonder-TinkhamKlapwijk theory; ${ }^{23}$ and $T_{3}=-(\Delta /|E|)\left[\Gamma_{L} \mathbf{G}_{11} \Gamma_{R} \mathbf{G}_{12}^{\dagger}+\right.$ H.c. $]$ describes the Cooper pair formation (or annihilation) inside the superconducting lead by an incoming electron (or hole). Clearly, at zero temperature when bias voltage $|V|>\Delta$, all the processes will contribute to current, while for $|V|<\Delta$ only the Andreev current $I_{A}$ is nonzero. 


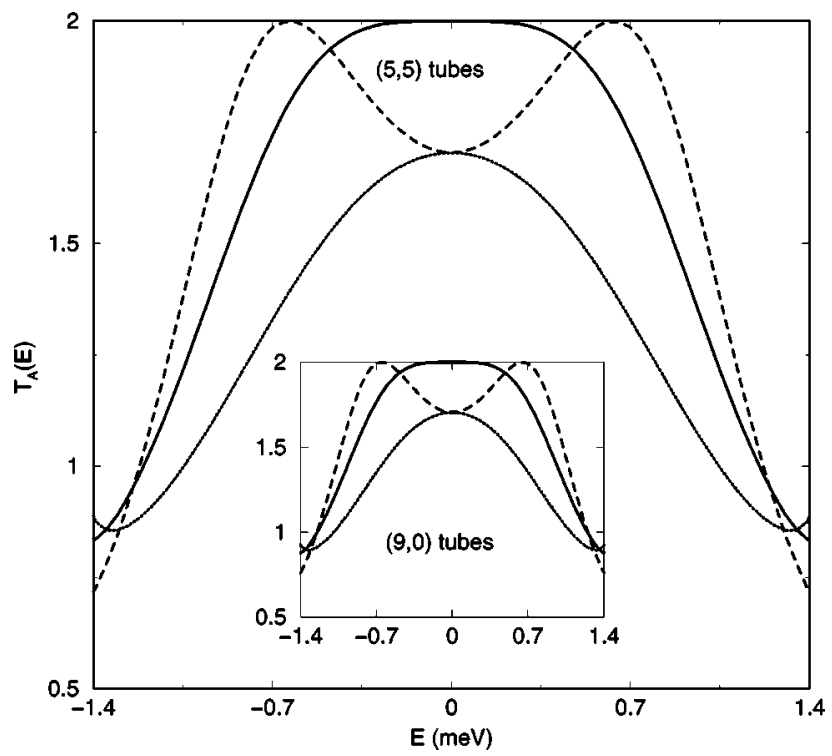

FIG. 1. Andreev reflection coefficient $T_{A}$ as a function of electron energy $E$. Main graph: for devices consisting of $(5,5) \mathrm{SWNT}$ with length $L=19$ unit cells. Inset: For $(9,0)$ zigzag nanotube systems. Other parameters are fixed as zero bias and zero gate voltages. Solid curve: $\Gamma_{L}=\Gamma_{R}=5 \times 10^{-3}$; dotted curve: $\Gamma_{L}$ $=6 \times 10^{-3}, \quad \Gamma_{R}=4 \times 10^{-3} ;$ dashed curve: $\Gamma_{L}=4 \times 10^{-3}, \quad \Gamma_{R}$ $=6 \times 10^{-3}$. Here the $\Gamma^{\prime}$ s are measured in a.u.

In our numerical calculations, we fixed the gap energy at $\Delta=1.45 \mathrm{meV}$ corresponding to that of Nb leads; the SWNT TB Hamiltonian is taken to be a nearest-neighbor $\pi$-orbital model with bond potential $V_{p p \pi}=-2.75 \mathrm{eV}$, which is known to give a reasonable description of the electronic and transport properties of carbon nanotubes. ${ }^{20,24} \mathrm{We}$ focus on metallic nanotubes of finite length $L$. The proximity of the nanotube to the superconductor is modeled through the coupling parameters $\Gamma_{L, R}$, which are treated as input parameters. $^{25}$

The solid lines of Fig. 1 show the Andreev reflection coefficient $T_{A}(E)$ for a metallic $(5,5)$ SWNT device of length ${ }^{26}$ $L=3 \times 6+1=19$, and for a $(9,0)$ zigzag SWNT device of $L=49$, with coupling parameter $\Gamma_{L}=\Gamma_{R}=5 \times 10^{-3}$ a.u. (1 a.u. energy is $13.6 \mathrm{eV})$. It is clear that resonant transmission with $T_{A}(E)=2.0$, i.e., high device transparency, dominates the transport at $E=0$. This may be understood as follows. It is well known, that infinitely long armchair nanotubes have two states crossing the Fermi level at $E_{F}=0$. On the other hand, a finite-length isolated armchair nanotube has a gap between the two eigenstates near $E_{F}$, and this gap is minimized for tube length $L=3 n+1$ where $n$ an integer. ${ }^{27,22}$ Therefore, when coupled to the device electrodes, which also adds a finite width to the levels, these nanotubes have two scattering states at $E_{F}$ giving rise to large transmission with $T_{A}=2$ as shown in Fig. 1. The large $T_{A}$ for the zigzag tube is also due to a resonance transmission through the nanotube states at the Fermi level. The two other curves in Fig. 1 show results for asymmetric couplings and will be discussed later. Thus, for a $N$-SWNT-S system, the device transparency is critically determined by quantum resonance phenomenon, which is qualitatively different from the case of an infinite

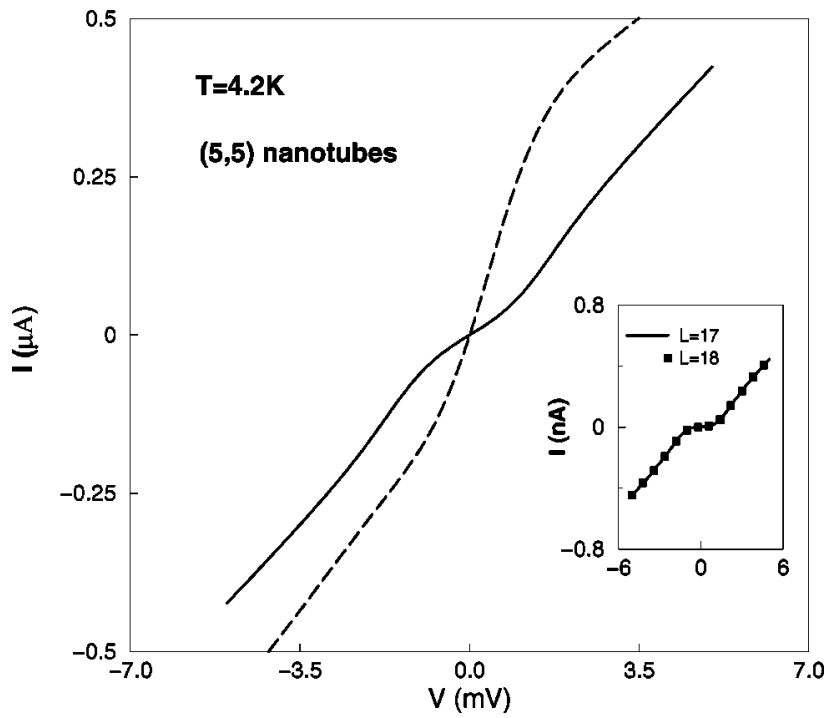

FIG. 2. $I-V$ curves for the $N$-SWNT-S device at temperature 4.2 $\mathrm{K}$. The SWNT is a $(5,5)$ metallic tube with length $L=19$ unit cells. Solid curve is for a low-transparency device (off-resonance transmission) with parameters $\Gamma_{L}=\Gamma_{R}=0.8$ and $V_{g}=0$. Dashed curve is for a high-transparency device (on-resonance transmission) with $\Gamma_{L}=\Gamma_{R}=5 \times 10^{-3}$ and $V_{g}=0.6 \mathrm{mV}$. Inset: $I-V$ curves (same units as the main graph) for tubes with $L=17$ and 18 unit cells with $\Gamma_{L}$ $=\Gamma_{R}=5 \times 10^{-3}$ and $V_{g}=0.6 \mathrm{mV}$.

SWNT. As expected, high-transparency devices have a larger $T_{A}$, since more electrons arriving at the SWNT-S interface increase the magnitude of the Andreev reflection process.

Figures 2 and 3 show the current-voltage characteristics, and the differential resistance $d V / d I$ for high- and lowtransparency $(5,5)$ SWNT devices, respectively. The $I-V$ curves of Fig. 2 are qualitatively consistent with and quantitatively close to the experimental data. ${ }^{4}$ It is clear that a higher slope is observed for the $I-V$ curves within the subgap range for on-resonance devices (dashed curve). This gives rise to an asymmetric resistance dip near bias voltage $V=0$ [see Fig. 3(a)] with the asymmetry due to the finite gate voltage $V_{g}$. This resistance dip is simply a reflection of the high value of $T_{A}$ for on-resonance devices (solid line, Fig. 1). The resistance dip has a value close to $h /\left(2 \times 4 e^{2}\right)=3.2 \mathrm{k} \Omega$, which is precisely the expected value of Andreev reflection processes in a SWNT-S junction with two transmitting modes. The experimental data ${ }^{4}$ for two SWNT-S junctions connected in series actually gives a value close to $5.7 \mathrm{~K} \Omega$, which is not far from the expected value of $6.4 \mathrm{~K} \Omega$. This difference is perhaps due to parallel connection of three SWNT's bridging the superconductor electrodes in the experimental setup. ${ }^{4}$ When the device transparency is low, the differential resistance displays a large peak at $V=0$, as shown in Fig. 3(b). This is consistent both with the $I-V$ curve of the low-transparency device shown in Fig. 2 and the experimental data. ${ }^{4}$

So far, the data presented have been for temperatures of $T=4.2 \mathrm{~K}$, so that features reflecting smaller energy scales are completely washed out. However, at a lower temperature of $T=2 \mathrm{~K}$, the experimental data ${ }^{4}$ shows that a narrow peak 


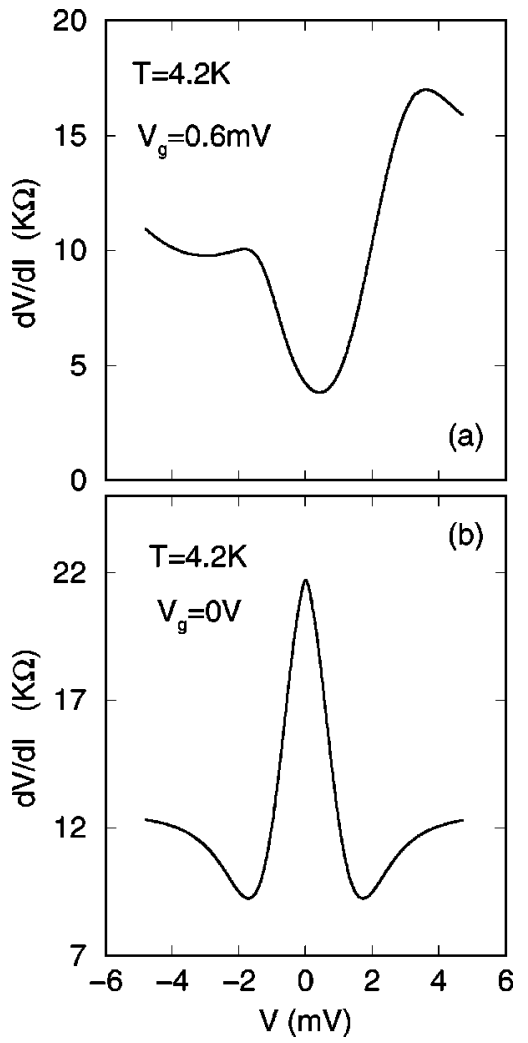

FIG. 3. Differential resistance $d V / d I$ as a function of bias voltage $V$. (a) For high-transparency (on-resonance) device corresponding to the dashed I-V curve of Fig. 2. (b) For low-transparency (off-resonance) device corresponding to the solid $I-V$ curve of Fig. 2. $(5,5)$ SWNT with length $L=19$ unit cells are used.

emerges in the $d V / d I$ curves for zero bias, which is superimposed on the Andreev dip. Such anomalous behavior has previously been ascribed to the strong electron-electron interactions characteristic of Luttinger liquids. Surprisingly, our analysis shows that these features emerge naturally at lower temperatures, even within the context of a singleelectron theory as presented here. This is shown in Fig. 4, which illustrates the emergence of a narrow peak out of the overall Andreev dip as the temperature is lowered.

We can trace this low-temperature anomaly to the basic physical process which gives rise to the Andreev current. To demonstrate this, we neglect complications due to the molecular structure of the nanotubes and assume that the resonant Andreev process is mediated by a single state at energy $E_{o}<\Delta$, i.e., we "shrink" the nanotube to a simple quantum well with a single level. This is qualitatively reasonable because the subgap energy scale is set by gap energy $\Delta$, which is much smaller than the level spacing of the nanotube we study ${ }^{28}$ Hence, we expect that only the two degenerate levels at the Fermi energy will contribute appreciably to the Andreev current. For this single-level case, the Green's functions are drastically simplified ${ }^{11}$ so that equivalently, familiar scattering matrix theory can be applied. ${ }^{6}$ Near the resonance, the transmission amplitude in the normal state assumes the Breit-Wigner form, $t(E)=i \sqrt{\Gamma_{L} \Gamma_{R}} /\left(E-E_{0}+i \Gamma / 2\right)$, and the reflection amplitude becomes $r(E)=1-i \Gamma_{R} /\left(E-E_{o}\right.$
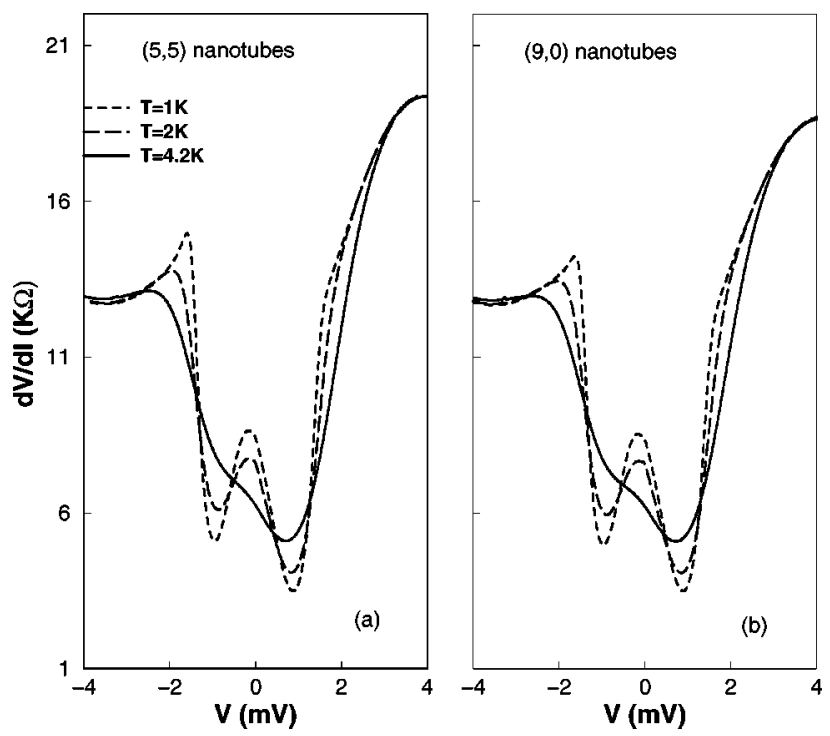

FIG. 4. Differential resistance $d V / d I$ for high-transparency devices at different temperatures. A peak emerges from the overall dip as temperature is lowered. (a) For $(5,5)$ nanotube devices with $L$ $=19$ unit cells. (b) For $(9,0)$ nanotubes with $L=49$. Other parameters: $V_{g}=0.6 \mathrm{mV}, \Gamma_{L}=3 \times 10^{-3}, \Gamma_{R}=8 \times 10^{-3}$.

$+i \Gamma / 2)$, where $\Gamma \equiv \Gamma_{L}+\Gamma_{R}$ is the total linewidth. For these amplitudes, Eq. (2) takes on the Breit-Wigner form:

$$
T_{A}(E)=\frac{\Gamma_{L}^{2} \Gamma_{R}^{2}}{4\left[E^{2}-E_{o}^{2}+\Gamma \delta \Gamma / 4\right]^{2}+\Gamma_{L}^{2} \Gamma_{R}^{2}+E_{o}^{2}\left[\Gamma^{2}+\delta \Gamma^{2}\right]},
$$

where $\delta \Gamma \equiv \Gamma_{L}-\Gamma_{R}$. This result allows us to draw several conclusions. For simplicity, we set the level at $E_{o}=0$, to obtain

$$
T_{A}(E)=\frac{\Gamma_{L}^{2} \Gamma_{R}^{2}}{4\left(E^{2}+\Gamma \delta \Gamma / 4\right)^{2}+\Gamma_{L}^{2} \Gamma_{R}^{2}} .
$$

These expressions reduce, exactly, to the result of scattering matrix theory for $N$-Dot- $S$ systems ${ }^{6}$ when we $\operatorname{set}^{29} E=E_{0}$ $=0$. Equation (5) indicates that if $\Gamma_{L}=\Gamma_{R}$ so that $\delta \Gamma=0$, then resonant Andreev reflection occurs at $E=0$ with $T_{A}(E$ $=0)=1$. On the other hand, if $\Gamma_{L}>\Gamma_{R}$ so that $\delta \Gamma>0, T_{A}$ takes on a maximum value at $E=0$ but this maximum value is less than 1. For nanotubes this situation is shown by the dotted lines of Fig. 1. Furthermore, if $\Gamma_{L}<\Gamma_{R}$ such that $\delta \Gamma$ $<0, T_{A}$ is characterized by two resonant peaks with $T_{A}=1$ at energies $E_{ \pm}= \pm \sqrt{-\Gamma \delta \Gamma} / 2$. For nanotubes this behavior is shown by the dashed lines of Fig. 1. Hence, due to a split between the electron and hole levels when the nanotube is in contact with a superconducting lead, the Andreev coefficient in the subgap region can display different behaviors. When $T_{A}(E)$ displays a double peak, the Andreev current $I_{A}$ shows a resistance anomaly such that a small peak develops inside the overall dip at low temperatures. At higher temperatures such as $4.2 \mathrm{~K}$, the anomaly is smeared out, and hence, not observable. One can also confirm that, qualitatively, the above conclusion holds for cases of nonzero gate voltage. 
The analysis presented so far has been for $(5,5)$ nanotubes with a length of $L=19$ unit cells and $(9,0)$ zigzag nanotubes ${ }^{26}$ with $L=49$. However, we expect our results to be general for other metallic nanotubes and lengths. Previous investigations showed ${ }^{22}$ that transport through finite armchair nanotubes differ qualitatively from the infinite length limit. In particular, if $L=3 n+1$ where $n$ is an integer, the armchair tube has large conductance due to the crossing of scattering state energy levels at the Fermi energy. Other tube lengths produce much smaller conductance due to a gap between the scattering states. The inset of Fig. 2 shows the $I-V$ curve of $N$-SWNT-S devices with $L=17$ and $L=18$ : these devices have very small currents because their device transparency are drastically diminished by the energy gap between the scattering states of the SWNT. Experimentally, such an energy gap along with conductance oscillations on finite tubes have already been detected with scanning probes. ${ }^{30}$

In summary, we have investigated the subgap transport properties of $\mathrm{N}$-SWNT-S systems and our results are consistent with a number of experimental observations. Specifically, the dependence of the Andreev current on the device transparency, the behavior of the differential resistance in the subgap region, as well as the observed low-temperature resistance anomaly may all be explained in terms of the proximity of the nanotubes with the superconducting lead. There are, however, still several issues which cannot be studied within the context of our model. First, the experimental data ${ }^{4}$ showed a sensitive gate voltage dependence at very low temperatures on the order of $40 \mathrm{mK}$, where the differential resistance anomaly could be a peak or a dip depending on the value of the gate voltage. This behavior has been considered as likely due to electron-electron interactions. ${ }^{4}$ Second, there is experimental and theoretical evidence that nanotubes can have non-Fermi-liquid behavior. ${ }^{31,32}$ It will be of great interest to investigate the situation of a non-Fermi-liquid model of nanotubes in contact with a superconductor to see if other, finer features emerge. Another important problem is the detailed atomic structural analysis of the nanotubesuperconductor interface, which, to a large extent, controls the interface transparency. Finally, although we do not expect charge transfer to play a critical role in understanding the Andreev current for SWNT-S interfaces because of the superconducting gap, a more complete investigation of this delicate effect will certainly enhance our understanding of quantum transport for nanoscale devices.

We gratefully acknowledge Professor T. H. Lin and Dr. Q. F. Sun for helpful discussions on the NEGF theory. We gratefully acknowledge financial support from NSERC of Canada and FCAR of Quebec (H.G.); a RGC grant (HKU 7215/99P) from the Hong Kong SAR (J.W.); and ONR N00014-98-1-0597 and NASA NAG8-1479 (C.R.). We also thank the North Carolina Supercomputing Center (NCSC) for significant amounts of computer time.
${ }^{1}$ Kazuhito Tsukagoshi, Bruce W. Alphenaar, and Hiroko Ago, Nature (London) 401, 572 (1999).

${ }^{2}$ Y. Zhang, T. Ichihashi, E. Landree, F. Nihey, and S. Iijima, Science 285, 1719 (1999).

${ }^{3}$ A. Yu. Kasumov, R. Deblock, M. Kociak, B. Reulet, H. Bouchiat, I. I. Khodos, Yu. B. Gorbatov, V. T. Volkov, C. Journet, and M. Burghard, Science 284, 1508 (1999).

${ }^{4}$ A. F. Morpurgo, J. Kong, C. M. Marcus, and H. Dai, Science 286, 263 (1999).

${ }^{5}$ A. F. Andreev, Zh. Éksp. Teor. Fiz. [Sov. Phys. JETP 19, 1228 (1964)].

${ }^{6}$ For reviews, see, for example, the article and references therein by C. W. J. Bennakker, in Mesoscopic Quantum Physics, Les Houches, Session LXI, 1994, edited by E. Akkermans, G. Montambaux, J. L. Pichard, and J. Zinn-Justin (Elsevier Science, 1995); Rev. Mod. Phys. 69, 731 (1997).

${ }^{7}$ C. J. Lambert and R. Raimondi, J. Phys.: Condens. Matter 10, 901 (1998).

${ }^{8}$ C.W. J. Beenakker, Phys. Rev. B 46, 12841 (1992); N. R. Claughton, M. Leadbeater, and C. J. Lambert, J. Phys.: Condens. Matter 7, 8757 (1995).

${ }^{9}$ S. Ishizaka, J. Sone, and T. Ando, Phys. Rev. B 52, 8358 (1995).

${ }^{10}$ A. L. Yeyati, A. Martin-Rodero, and J. C. Curvas, J. Phys.: Condens. Matter 8, 449 (1996); Phys. Rev. B 54, 7366 (1996); A. L. Yeyati, J. C. Cuevas, A. Lópec-Dávalos, and A. Martin-Rodero, ibid. 55, R6137 (1997).

${ }^{11}$ Qing-feng Sun, Jian Wang, and Tsung-han Lin, Phys. Rev. B 59, 3831 (1999).
${ }^{12}$ E. N. Bratus', V. S. Shumeiko, and G. Wendin, Phys. Rev. Lett. 74, 2110 (1995); V. S. Shumeiko, E. N. Bratus, and G. Wendin, Low Temp. Phys. 23, 181 (1997); E. V. Bezuglyi, E. N. Bratus', V. S. Shumeiko, and G. Wendin, Phys. Rev. Lett. 83, 2050 (1999).

${ }^{13}$ N. van der Post, E. T. Peters, I. K. Yanson, and J. M. van Ruitenbeek, Phys. Rev. Lett. 73, 2611 (1994); B. Ludoph, N. van der Post, E. N. Bratus', E. V. Bezuglyi, V. S. Shumeiko, G. Wendin, and J. M. van Ruitenbeek, Phys. Rev. B 61, 8561 (2000).

${ }^{14}$ A. W. Kleinsasser, R. E. Miller, W. H. Mallison, and G. B. Arnold, Phys. Rev. Lett. 72, 1738 (1994).

${ }^{15}$ A. Kastalsky, A. W. Kleinsasser, L. H. Greene, R. Bhat, F. P. Milliken, and J. P. Harbison, Phys. Rev. Lett. 67, 3026 (1991); S. J. M. Bakker, H. M. Jaeger, T. M. Klapwijk, E. van der Drift, and S. Radelaar, Phys. Rev. B 48, 4168 (1993).

${ }^{16}$ E. Scheer, P. Joyez, D. Esteve, C. Urbina, and M. H. Devoret, Phys. Rev. Lett. 78, 3535 (1997).

${ }^{17}$ V. A. Khlus, A. V. Dyomin, and A. L. Zazunov, Physica C 214, 413 (1993).

${ }^{18}$ A. V. Demin, V. A. Khlus, and L. P. Chernyakova, Low Temp. Phys. 21, 413 (1995).

${ }^{19}$ I. L. Aleiner, P. Clarke, and L. I. Glazman, Phys. Rev. B 53, R7630 (1996).

${ }^{20}$ L. Chico, V. H. Crespi, L. X. Benedict, S. G. Louie, and M. L. Cohen, Phys. Rev. Lett. 76, 971 (1996); V. H. Crespi, M. L. Cohen, and A. Rubio, ibid. 79, 2093 (1997); L. Chico, M. P. Sancho, and M. C. Munoz, ibid. 81, 1278 (1998); L. Chico, L. 
X. Benedict, S. G. Louie, and M. L. Cohen, Phys. Rev. B 54, 2600 (1996).

${ }^{21}$ A. P. Jauho, N. S. Wingreen, and Y. Meir, Phys. Rev. B 50, 5528 (1994).

${ }^{22}$ H. Mehrez, J. Taylor, H. Guo, J. Wang, and C. Roland, Phys. Rev. Lett. 84, 2682 (2000).

${ }^{23}$ G. E. Blonder, M. Tinkham, and T. M. Klapwijk, Phys. Rev. B 25, 4515 (1982).

${ }^{24}$ M. Buongiorno Nardelli, Phys. Rev. B 60, 7828 (1999); A. Rochefort, P. Avouris, F. Lesage, and D. Salahub, ibid. 60, 13824 (1999).

${ }^{25}$ See, for example, $a b$ initio calculations for normal metal leads: C. C. Wan, José-Luis Mozos, Gianni Tarachi, Jian Wang, and Hong Guo, Appl. Phys. Lett. 71, 419 (1997); Phys. Rev. B 55, R13 393 (1997); José-Luis Mozos, C. C. Wan, Gianni Taraschi, Jian Wang, and Hong Guo, ibid. 56, R4351 (1997); Gianni Taraschi, J.-L. Mozos, C. C. Wan, Hong Guo, and Jian Wang, ibid. 58, 13138 (1998); Jian Wang, Hong Guo, Jose-Luis Mozos, C. C. Wan, Gianni Taraschi, and Qingrong Zheng, Phys. Rev. Lett. 80, 4277 (1998).

${ }^{26}$ For armchair nanotubes $L$ is measured in terms of unit cells: a unit cell is the repeat unit along the armchair tube consisting of two carbon rings. For zigzag nanotubes $L$ is in terms of the number of carbon rings.

${ }^{27}$ A. Rochefort, D. R. Salahub, and P. Avouris, J. Phys. Chem. B 103, 641 (1999).

${ }^{28}$ The energy level spacing along the finite single wall tube can be estimated from the one-dimensional particle-in-a-box model [see Angel Rubio, Daniel Sanchez-Portal, Emilio Artacho, Pablo Ordejsn, and Josi M. Soler, Phys. Rev. Lett. 82, 3520 (1999)] to be
$\Delta E=(1.68 \mathrm{eV} \mathrm{nm}) / \mathrm{L}$, where $L$ is the tube length. The relevant energy scale of our problem (the subgap region where Andreev physics dominant) is $\Delta=1.45 \mathrm{meV}$, hence we estimate that the level spacing becomes important for our problem when tube length $L$ approaches $1000 \mathrm{~nm}$. For the shorter nanotube devices examined in this work, we therefore neglect the higher-energy levels of the nanotube in the arguments for understanding the transport anomaly.

${ }^{29}$ One can easily confirm that the Eq. (4) or (5), which were obtained from the Green's function theory reduces to exactly the same result as scattering matrix theory of Ref. 6, [e.g. Eq. (253) of Rev. Mod. Phys. 69, 731 (1997)] for the conductance of NINIS systems by setting $E=0$ and $V=0$, which were the assumptions made in the scattering matrix derivations. Here $N$ stands for normal, $I$ for insulating, and $S$ for superconducting materials. In particular, our result for conductance obtained from $T_{A}$ of Eq. (4) or (5) is exactly the same as given by Eq. (3.8) of Ref. 6 if we set $E=0$ in our expressions.

${ }^{30}$ L. C. Venema, J. Wildoeer, J. Janssen, S. J. Tans, H. Temminck Tuinstra, L. Kouewenhovem, and C. Dekker, Science 283, 52 (1999).

${ }^{31}$ Marc Bockrath, D. H. Cobden, J. Lu, A. G. Rinzler, R. E. Smalley, L. Balents, and P. L. McEuen, Nature (London) 397, 598 (1999); Z. Yao, H. Postma, L. Balents, and C. Deeker, ibid. 402, 273 (1999); Reihold Egger and Alexander O. Gogolin, Phys. Rev. Lett. 79, 5082 (1997); Charles Kane, Leon Balents, and Matthew P. A. Fisher, ibid. 79, 5086 (1997).

${ }^{32}$ M. Bockrath, D. H. Cobden, P. L. McEuen, N. G. Chopra, A. Zettl, A. Thess, and R. E. Smalley, Science 275, 1922 (1997). 